\title{
Qarn Alam: Application of Simulation for Steam Pilot Design in a Fractured Reservoir
}

\author{
* Hartemink M., * Escovedo B. M., ** Hoppe J. E., ** Macaulay R
}

\author{
* Shell, The Netherlands \\ ** Petr. Dev. Oman, Oman
}

Copyright 1986, Steering Committee of the European IOR - Symposium.

This paper was presented at the Oth. European IOR - Symposium in Vienna. Aubtria, May 16 - 17. 1995

This paper was selected for presentetion by the Steering Committes, following roview of informetion contained in an abstract

aubmitted by the author(s). The paper, as presented has not been reviewed by the stearing committee.

\begin{abstract}
Late in 1995 a steam-injection pilot project will be initiated in the Qarn Alam field in Oman. The objective is to test the steam-enhanced gas-oil gravity drainage of the heavy oil remaining in the matrix of the fractured carbonate Shuaiba reservoir. This paper presents the results of reservoir simulations that were conducted to investigate the design and future interpretation of the steam pilot. Different simulation techniques have been appiied to describe the complexity of thermal processes and multiphase flow in a densily fractured reservoir. Two models, both using SSI's THERM reservoir simulator, have been employed. With the first, a 3D element-of-symmetry sector model, pilot performance was investigated, using explicit modelling of the fractures. A second 3D model (which only considered flow in the fractures) was created to investigate the areal advance of the steam zone, given a certain fracturepermeability anisotropy, fracture spacing and reservoir structure. The possibility of oil rim lowering, prior to injecting steam, and of injecting steam in two different local crests, were also investigated but rejected on basis of simulation results. Finally, locations for steam injection wells and pilot observation wells are proposed and requirements for pilot monitoring deduced.
\end{abstract}

\section{INTRODUCTION}

The Qarn Alam field in north Oman contains a fractured carbonate reservoir characterised by densely spaced, high-permeability fractures (ranging from $1 \mathrm{D}$ to 1000 D) running through a relatively low-permeability matrix (of approximately $5-15 \mathrm{mD}$ ), which is thought to be oil-wet. The structure is a folded anticline and is therefore commonly referred to as dome shaped. The stock-tank oil initially in place (STOIIP) is estimated to be 213 million $\mathrm{m}^{3}$, and the in-situ oil viscosity at the original reservoir temperature is estimated at $220 \mathrm{mPa}$.s. To date, only some $2 \%$ of the total STOIIP has been recovered, most of which has come from the fracture system.

The main mechanism of oil recovery has been an active water drive in the fractures; it is responsible for the substantial percentage of the total primary oil that was recovered during the first years of the field's production. What remained after the initial production was a small oil rim some $30 \mathrm{~m}$ thick in the fracture system which is sandwiched between a $30-\mathrm{m}$ fracture (secondary) gas cap above and the invaded aquifer water below.

Recent modelling has confirmed the likelihood of gas-oil gravity drainage from the $30 \mathrm{~m}$ of matrix adjacent to the secondary gas cap above the oil rim and water-oil gravity drainage below the oil rim (some 
additional $150 \mathrm{~m}$ ). Owing to the oil-wet matrix, spontaneous imbibition below the oil rim is thought to be negligible. The current active recovery mechanisms are very slow and it is estimated that $98 \%$ of the STOIIP still remains within the low-permeability matrix.

In order to determine whether the gas-oil gravity drainage rate from the matrix to the fractures can be economically accelerated, a pilot project is planned in which steam will be injected into the fracture secondary gas cap in the crestal area of the Qarn Alam field. This paper discusses the results of numerical modelling of the steam pilot. Offtake scenarios and suitable locations for steam-injection and observation wells are presented. These aspects of the pilot's design were based on the expected shape and rate of advance of the steam zone and the anticipated pilot response in the production wells.

\section{SIMULATION MODELS}

\section{Sector model}

The first of the two models that were created for the study is a dual-porosity sector model. This model, which assumes that the reservoir has radial symmetry, consists of a cartesian $72 \times 2 \times 15$ grid structure that explicitly describes one fracture, of $0.6 \mathrm{~mm}$ width, adjacent to a matrix block that is $0.30 \mathrm{~m}$ wide. The effective permeability of the model has been arbitrarily set at $40 \mathrm{D}$. The pore volume, transmissibility and heat capacity of the gridblocks have been adjusted in order to describe the radial element of symmetry as shown in Fig. 1 .

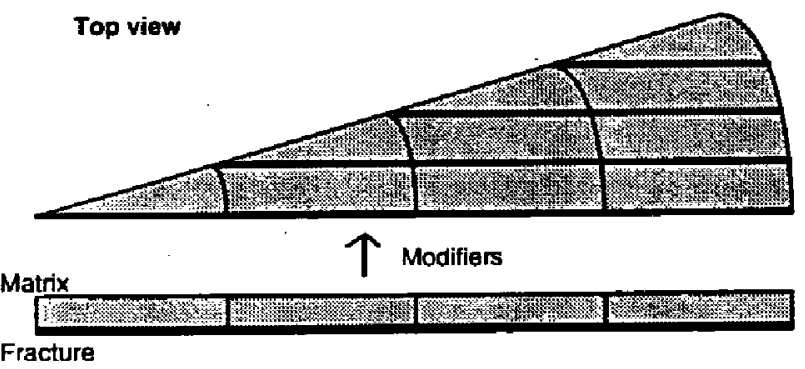

Cross-section

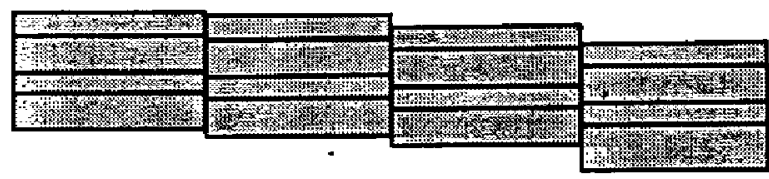

Figure 1 Qarn Alam sector model. showing only four layers and four radial blocks. The model consists of one explicit fracture adjacent to the marrix (centre). (Modifiers were used to represent the sector shown in top view (top)). The cross sectional view (below) shows the reservoir dipping away from the crest.
The element encompasses a $0.57^{\circ}$ angle of the field aind conforms to the variable dipping nature of the structure (mild at the crest to steep on the flanks). The model's steam injector is placed at the top of the structure and in the centre of the field; its completion lies in a fracture gridblock in the secondary gas cap. The model's producer is located $270 \mathrm{~m}$ from the injector, and its completion is also in a fracture gridblock but deeper than that of the injector (in the oil rim).

\section{Crestal fracture model}

Realising that the sector model could not be used to investigate areal effects, we developed another 3D model that incorporates areal details of the structure. With this model, we could study the effect of fracture anisotropy on the shape of the steam zone. This model incorporates the azimuthal structure of the- field, as derived from recently acquired $3 \mathrm{D}$ seismic data. The data showed for the first time the existence of two local crests at the top of the structure as opposed to the idealised dome shape assumed in the sector model.

The crestal fracture model takes only a single porosity into account; in fact, it includes only the fracture characteristics. The matrix has been modelled as nonporous; it was included as such only to properly model the heat balance. Using this model with the correct position of the fracture gas cap and the fracture oil rim as input, we were able to simulate the areal development of the steam zone and to investigate the sensitivities to structure and fracture anisotropy. Because of the large number of gridblocks required for areal modelling, CPU times were correspondingly large. The model was therefore used only to investigate sensitivities for the first years of the steam-injection pilot.

\section{MODELLING RESULTS}

\section{Primary history match}

Our first step was to complete a match of the primary production history of the field utilising the sector model. As shown in Fig. 2, using actual historical gross production as input, the model accurately matched the primary oil, water production and the pressure behaviour. It was considered critical to match the water cut at both early time and after 5000 days, since the former gave us an indication of the aquifer strength and the latter gave us a measure of the primary gravitydrainage rate.

It appears that, in the fractures of this particular system, the oil rim naturally migrates to the highest próducing completion, maintaining a gas cap above and a water leg below. The position of the oil rim, in turn, determines the oil rate of the system, since it controls the amount of gravity drainage from the matrix to the fracture system. 


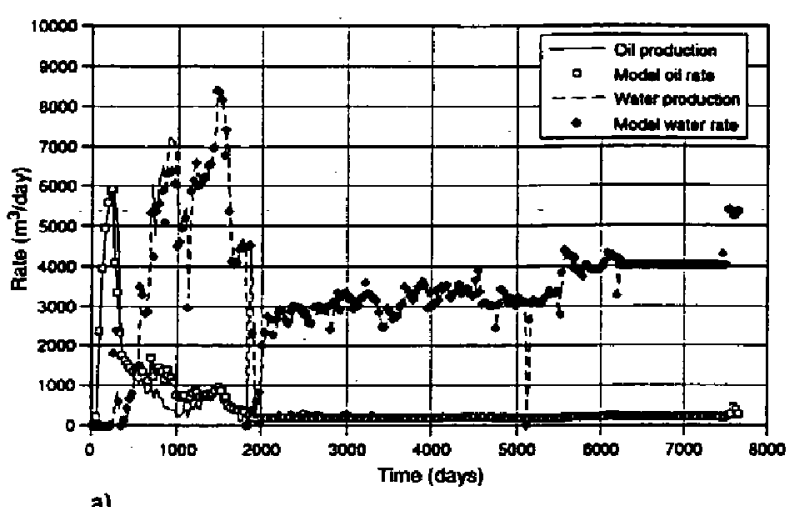

a)

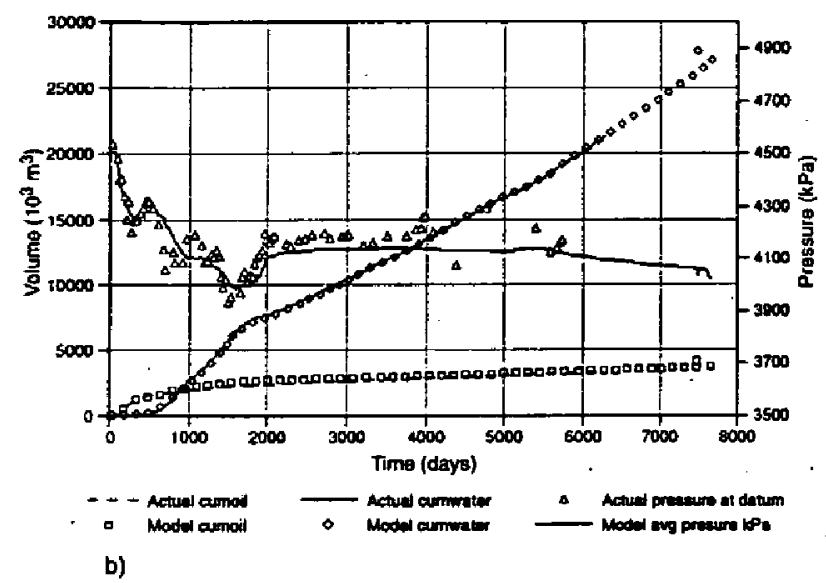

Figure 2 Qam Alam primary hissory match with the sector model. The historical oil and water rates (a) and the match of the cumulative production of oil, water and historical pressure $(b)$ are shown.

\section{Pilot saturation and temperature distribution}

After matching the first 7700 days of primary production history (Fig. 2), we introduced the coldwater equivalent (CWE) of $1000 \mathrm{~m}^{3} /$ day of steam into the model, to begin making predictions about the pilot. The production well gross constraint was simultaneously increased by $1000 \mathrm{~m}^{3 /}$ day, to 5500 $\mathrm{m}^{3} /$ day, to accommodate the incremental injection volume. Figure $3 a$ displays the predicted saturation distribution through time for the duration of the steam pilot. As can be seen, near the steam injector (in the top left corner of the figure), the oil rim quickly begins to thicken as the oil viscosity in the matrix is lowered and gas-oil gravity drainage from the matrix to the fracture system begins to accelerate. The oil rim then progresses in the direction of the producer and the producer begins to respond. Note that only a minor expansion of the gas cap in the vertical direction occurs, as the oil rim is being kept level (no pressure change).

Figure $3 b$ displays the temperature distribution during the pilot. It shows that after steam injection has commenced, a high-temperature steam zone approximately $30 \mathrm{~m}$ thick invades (somewhat like a piston) the secondary gas in the fractures. The pistonlike displacement of the secondary gas by the steam is encouraged by the high velocity of steam in the fractures (which creates high viscous forces) and the resistance of the cold viscous oil rim in the fractures (which prevents the steam from displacing the oil downwards).

\section{Pilot production response and sensitivities}

The pilot oil-production response is shown in Fig. 4 for several different cases. The "cold oil rate" case is a continuation of. the primary history match and represents the baseline by which the incremental thermal oil rate was determined. The steam-injection sensitivity cases modelled include:

1) "Gross rate $7500 \mathrm{~m}^{3} /$ day" - an increased offtake rate;

2) "Steam Inj Base Case" - the pilot base case;

3) "Perm 5 mD" - a reduced matrix permeability;

4) "Distance $370 \mathrm{~m}$ " - an increased producer-injector spacing;

5) "Two inj. locations" - two injectors $500 \mathrm{~m}$ apart.

With the exception of the "Perm $5 \mathrm{mD}$ " case, in which the matrix permeability is assumed to be homogeneous $\left(k_{\mathrm{y}}=\mathrm{k}_{\mathrm{h}}\right)$ but low, the production response of the steam pilot is very similar for all cases. All cases additionally exhibited an increase in the volume of the oil rim. As discussed above, when the heated oil starts draining from the matrix by accelerated gas-oil gravity drainage, it is not immediately captured by the producing well. As a consequence, the oil rim grows in size to accommodate the draining oil.

The temperature and saturation distributions were also similar for all cases, except for those observed in an additional sensitivity case run with a reduced density difference between oil and fresh water (not shown). In this case, a mixing zone of oil and condensed water developed in the fracture oil rim and grew much larger than the segregated oil rim shown in Fig. 3a. The density difference between oil and water at temperatures around $220^{\circ} \mathrm{C}$ is currently being investigated in view of its potential influence on the steam pilot's behaviour.

Finally, a comparison of the simulation results was made with prior semi-analytical modelling done at Shell Research Rijswijk [1]. It was found that if the observed growth of the oil-rim volume, which amounts to some $100 \mathrm{~m}^{3} /$ day, is added to the observed production, the pilot response comes into good agreement with the steam-pilot predictions from the analytical modelling. 
Fraction Oil Saturation: Ternary
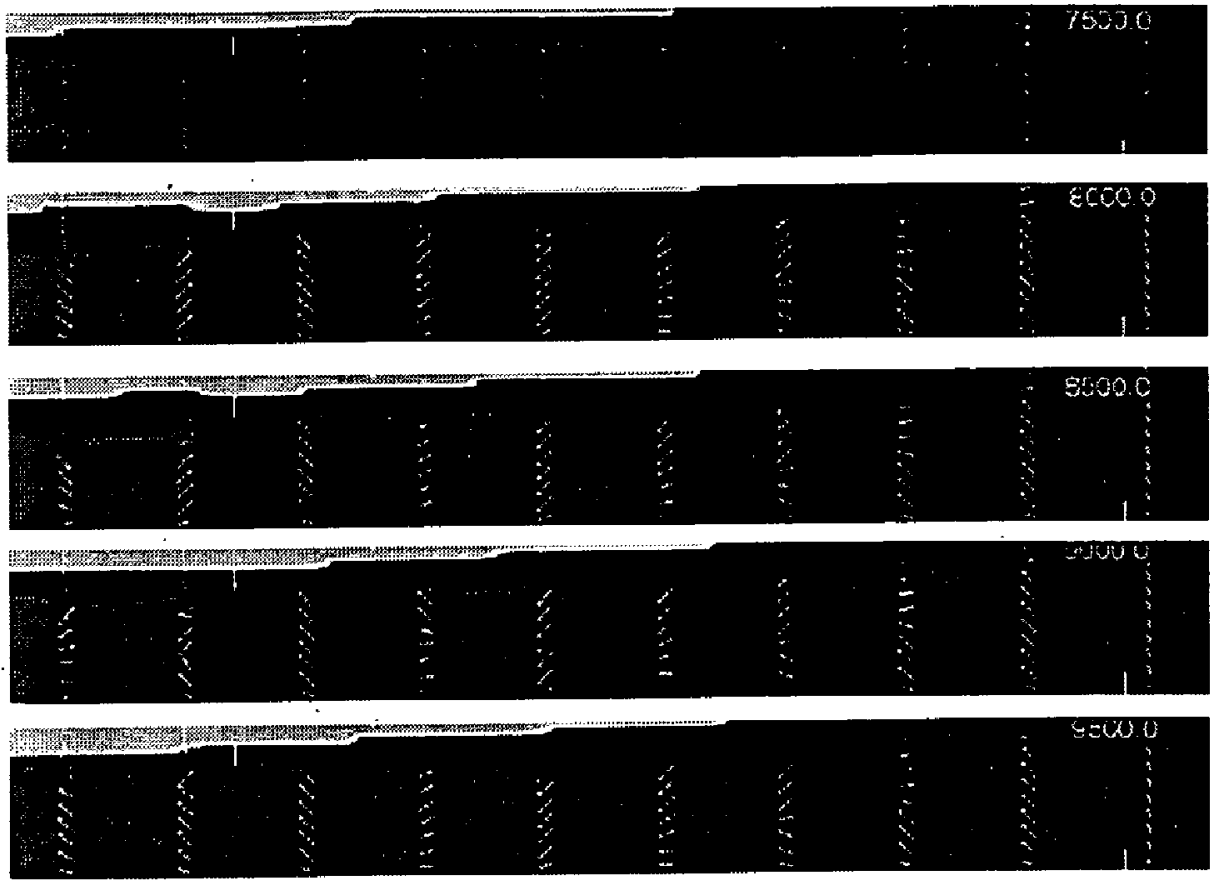

Fracture Temperature (50-234 degr. C)
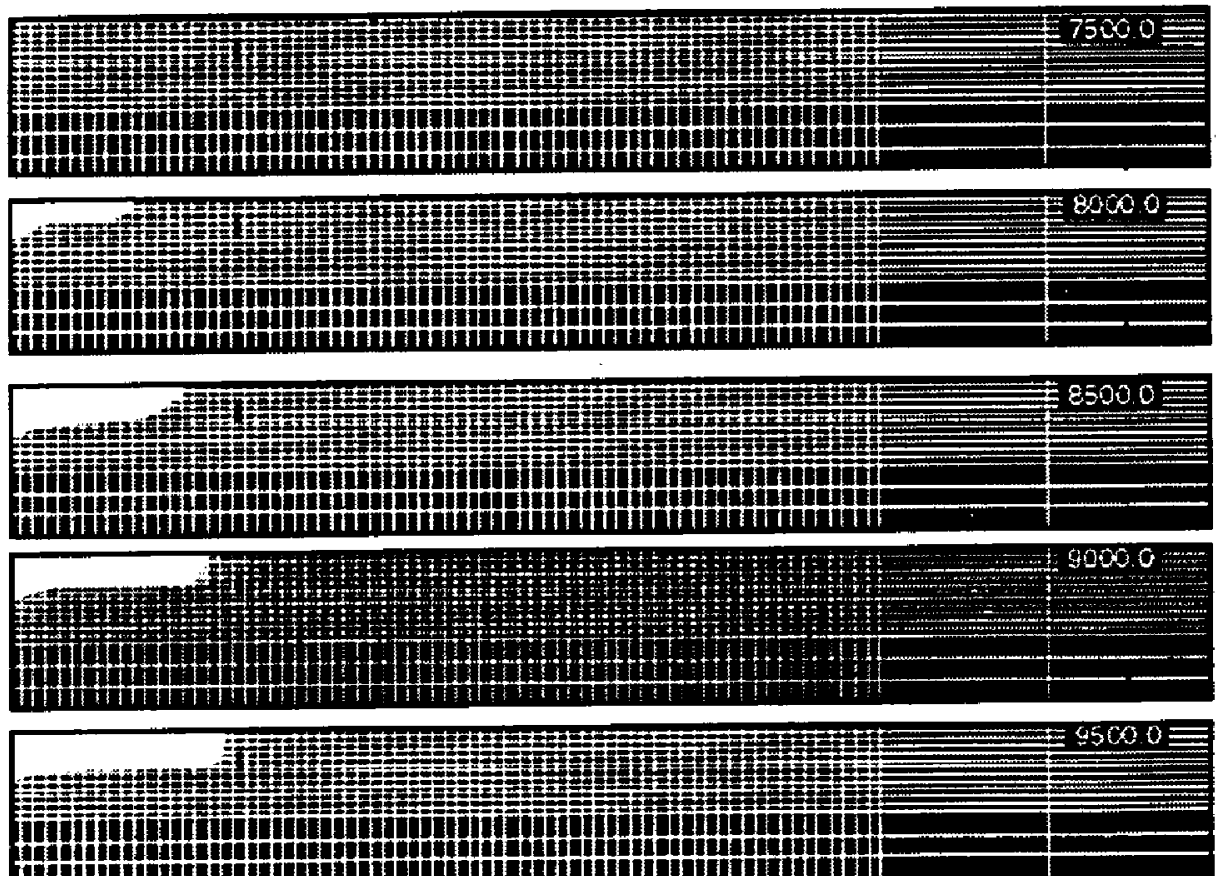

3b)

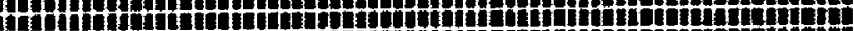

Figure 3 Fracture saturation ( $a$ ) and temperature distribution (b) as predicted by the sector model.

The time in days is indicated in the top right hand comer. Steam injection was started 7700 days into the simulation. In Fig. 3a the oil rtm (black) is sandwiched between the water leg (dark grey) and the gas cap (light grey). In Fig. 36 the steam zone (white) has a very steep temperature gradient to the cald resenvir. Note that the gridblocks (shown in Fig. 3 b) are actually dipping which is reflected in the oil rim. 
The analytical modelling was based on the assumption that the fracture system had no storage capacity and thus all oil drained from the matrix would be immediately captured by the producing well.

\section{Steam zone cross-sectional shape}

In order to study the cross-sectional ( $x-z$ plane) shape of the steam zone in more detail, the sector model was discretized further to include a more refined grid at the crest. This refined model confirmed the observed 25-30 $m$ thickness of the steam zone seen in the unrefined model and additionally confirmed that a piston-like displacement would occur even when the effective permeability is as high as $400 \mathrm{D}$. For higher values of permeability, however, the steam zone shape seemed to change into that characteristic of steam override as the steam progressed away from the injector.

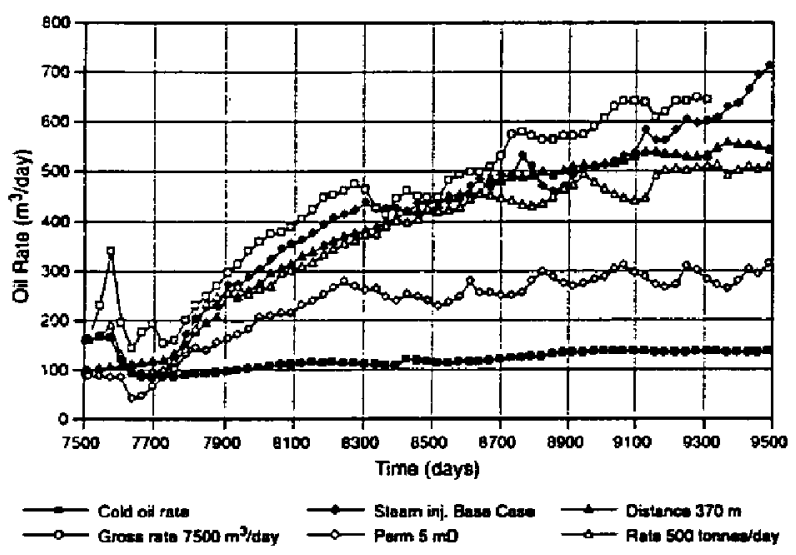

Figure 4 Predictions of the oil production rate for different scenarios for the steam pilot.

Because of the uncertainty in the fracture permeability, in the gas-steam density difference and in the degree of fracture anisotropy, the cross-sectional shape of the steam zone remains uncertain. However, one can reasonably suppose that the true shape of the steam zone will lie in between the two extreme cases, piston-like displacement and steam override. That supposition is enough to provide some aid in the design of the pilot and the placement of the injection and observation wells.

\section{i) piston-like displacement of steam zone}

The radial symmetry of the steam zone, implicitly assumed in the sector model, was confirmed by the simulations with the crestal fracture model (shown in Fig. 5). Given that the steam zone will develop radially and will be piston-like in cross-section, the radius $r$ of the steam zone development can be plotted as a function of time $t$ for two injection rates as shown in Fig 6a.
For an injection rate of 1000 tonnes of CWE steam per day, the curve in Fig. 6a can be approximated by

$$
r(m)=6.0 \sqrt{t(\text { days })}
$$

The relative error in Eq. [1] is less than 15\%, at least for the time during which the pilot will be conducted (fewer than 1800 days). Note that it takes 1740 days of continuous injection before the steam zone reaches a radius of $250 \mathrm{~m}$. Because the producing wells are further than $250 \mathrm{~m}$, steam breakthrough is unlikely to be observed in the production wells during the 5-year life of the steam pilot.

When the injection rate is lowered to 500 tonnes of CWE steam per day, the steam zone expands much more slowly. The corresponding curve in Fig. 6a can be approximated by the following equation:

$$
r(m)=4.2 \sqrt{t(\text { days })}
$$

The relative error here is also less than $15 \%$. As one would expect, the associated area is approximately half the area for the case in which the injection rate was 1000 tonnes/day. As a consequence, the response of the existing producing wells to steam injection with two wells at a rate of 500 tonnes/day is expected to be slightly weaker because of the slightly higher heat losses.

\section{ii) steam override}

The extreme opposite of a piston-like displacement is instantaneous steam override. The position of a horizontal gravity-stable steam front (associated with override) through time can be easily determined using the total heated reservoir volume in the predictions of the sector model. Reading the corresponding depth from the volume-versus-depth relationship of the structure map, one can plot the depth of the steam zone as a function of time. Figure $6 b$ shows the vertical position of the condensation front versus time using the structure map based on 3D seismic data. The condensation front as predicted in previous analytical modelling [1] is also shown. However, these results are based on a previous structural interpretation that included a smailer rock volume at the crest.

\section{Fracture spacing}

The previous results are valid under the assumption that, as the heat front progresses, the entire matrix adjacent to the fractures heats up at the same time as the fractures. In other words, the fractures are so dense that they expose an extremely high cross-sectional area to the heat, guaranteeing a virtually instantaneous heat transfer by conduction from the fractures to the matrix. This 


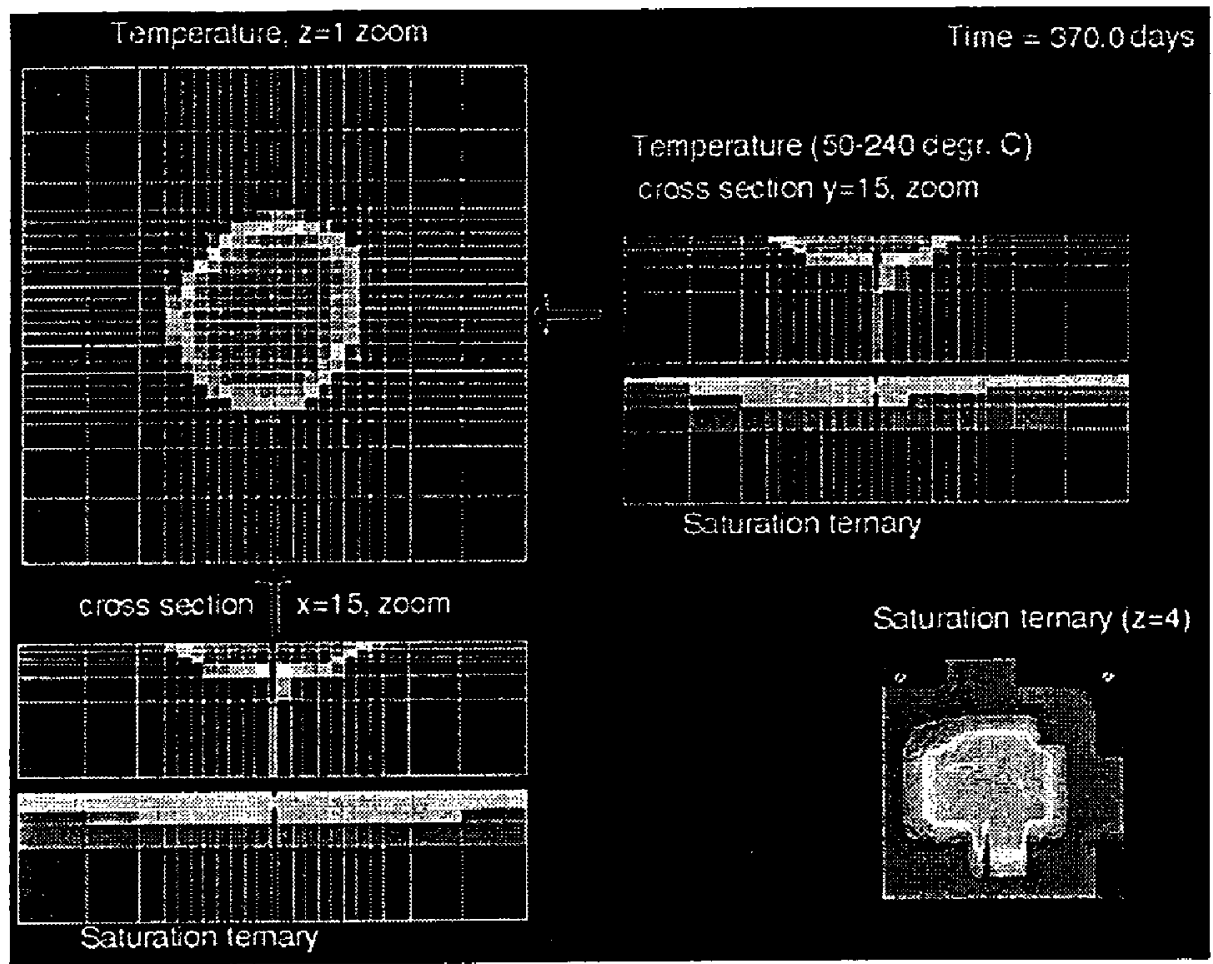

Figure 5 Temperature distribution (top left) as predicted by the crestal fracture model for the steam pilot. The saturation distribution (bottom right) and cross-sectional views for temperature and saturation distribution are also shown.

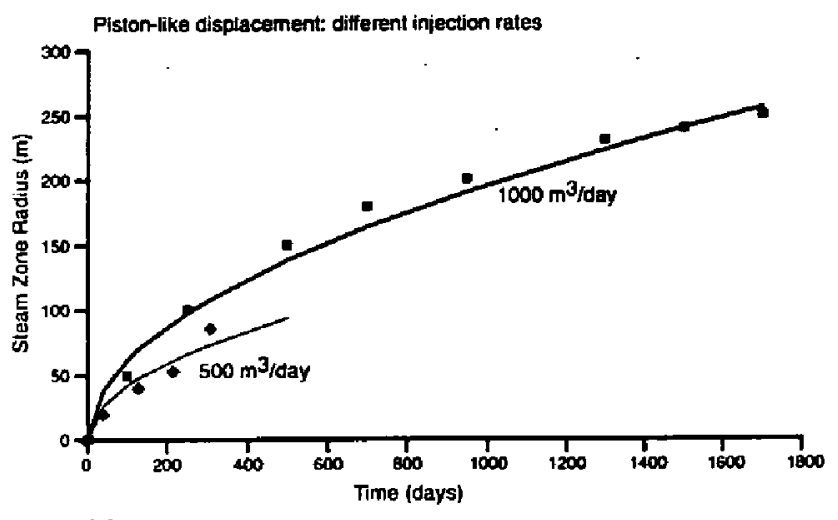

(a)

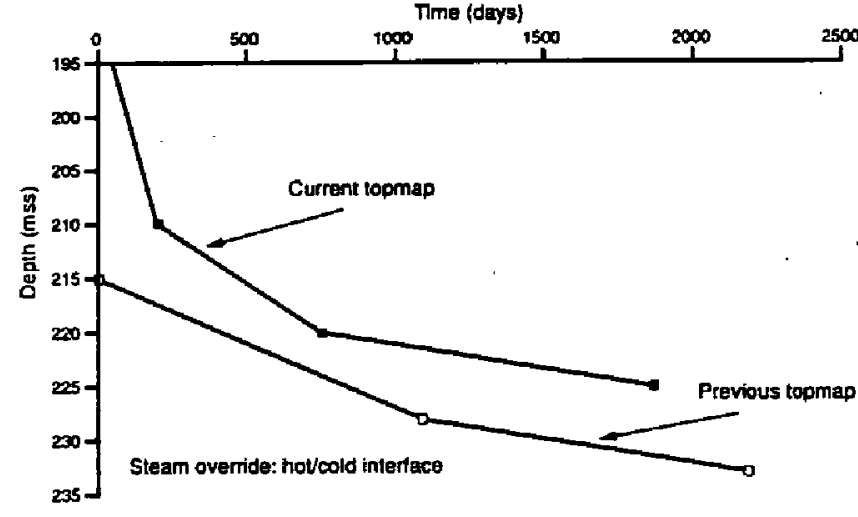

(b)

Figure 6 Predictions of radial growth for piston-like displacement on basis of the simulations (a) and predictions of the depth of the steam zone in the case of steam override (b)

assumption is quite reasonable for a fracture spacing as smail as $0.30 \mathrm{~m}$. However, as the fracture spacing increases, so too does the average size of the matrix blocks relative to the available contact area for heat transfer. At a certain point, heat transfer is no longer sufficient to heat the matrix blocks, and the steam advances in the fractures while the matrix heating lags behind. Regardless of fracture spacing, the total volume of heated rock will be the same, but its distribution will differ depending on the fracture spacing.

Although the thickness of the matrix blocks is never expected to exceed $3 \mathrm{~m}$ (on the basis of a recent fracture study), the fracture spacing remains uncertain in Qarn Alam. To study the sensitivity of our results to variations in fracture spacing, an investigation into the possible lag in matrix temperature was undertaken by taking the sector model and discretizing the matrix in the azimuthal direction into five blocks. It was found that, at a fracture spacing of $3 \mathrm{~m}$, the heat front at the center of the matrix between two fractures lagged the heat front in the fracture by approximately $15 \mathrm{~m}$. That means that there is a time lag of approximately 2-3 months before the entire matrix between the fractures is heated. The production response and the saturation 


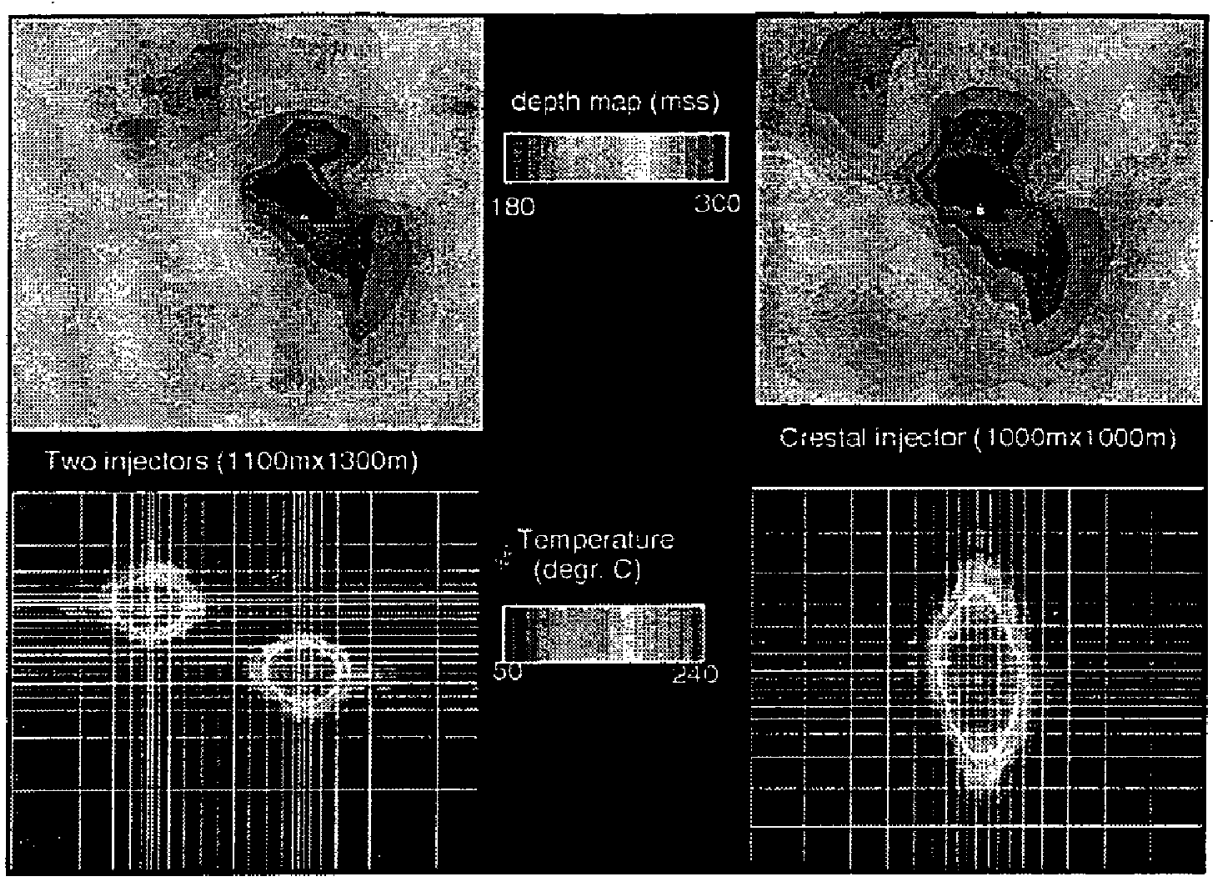

Figure 7 Temperature distribution after one year of steam injection. The left case shows two steam injectors at different locations, $500 \mathrm{~m}$ apart and the right case shows the impact of fracture anisotropy. Each are shown with their respective depth maps. For the case of fracture anisotropy, an effective fracture permeability of $400 \mathrm{D}$ in the NS and $40 \mathrm{D}$ in the EW direction were assumed, in line with the expected permeability trend.

distribution, however, were found to be similar to the predicted response for the $0.30 \mathrm{~m}$ fracture spacing. The reason is that the volume of heated rock actually remains constant. Hence, the same oil volume is being heated and therefore the production response should not vary much.

\section{Lowering the fracture oil rim prior to pilot}

In order for the steam zone to expand into the rest of the Shuaiba, it is anticipated that the oil rim will eventually have to be lowered. Although the original pilot design called for maintaining the oil rim in its current position throughout the pilot, it was decided to investigate the effects of lowering the oil rim prior to commencing steam injection. Such an action was thought to offer two hypothetical advantages: not only might it improve the primary gas/oil gravity drainage rate, but it might also produce a cleaner signal of the accelerated gas-oil gravity drainage once steam injection began.

Using the sector model, we discovered that, upon lowering the oil rim by lowering the completion and byincreasing the gross rate, the oil rim did not immediately re-establish itself at the deeper -structural position. Instead, it actually disappeared locally as the oil production rate from the fractures exceeded the improved drainage rate from the oil column, at least during the time period investigated. Also, upon commencing steam injection, the incremental oil response was less than the base case, because the steam zone in the fractures took on a different shape affecting the drainage rate from the matrix.

In contrast to prior results, the steam zone no longer had a resistant oil rim to keep it high in the fractures. With the oil rim lowered and out of the way, the steam zone penetrated vertically deeper into the enlarged gas cap in the fractures, reducing its radial extent. This reduced the heated horizontal cross-sectional area in the matrix and consequently led to a reduction in the thermal gas-oil gravity drainage rate.

\section{Two steam injectors in different local crests}

It is currently anticipated that two injectors will be needed to inject the $1000 \mathrm{~m}^{3} /$ day of CWE steam called for in the pilot design. New structure maps (Fig. 7) derived from 3D seismics indicate that there are two local crests at the top of the structure instead of just one as was previously assumed. The question was therefore raised as to the possibility of simultaneous injection of 500 tonnes/day through two wells, each on one of the local crests.

Using the sector model to model only one of the two local crests, we simulated the expected production response from the injection of a 500 tonnes/day injection of steam. As expected, the oil production response was less than $50 \%$ of the previous sector model, which was run at 1000 tonnes/day. The decrease in response was likely due to increased heat losses to the cap rock associated with injecting at two sites instead of one. 
Runs with the crestal fracture model with an injection well placed into both local crests additionally revealed that the steam zones are not likely to grow together during the five-year pilot. The temperature distribution after one year of injection is shown in Fig. 7.

Combining these results leads one to the conclusion that only one crest should be chosen for the pilot. This should maximise production response while minimising the amount of capital and operating expense, since one would not need to monitor two separate structures.

\section{Fracture anisotropy}

As some degree of anisotropy in fracture permeability is to be expected in the Qarn Alam reservoir, the impact of this effect was assessed with the crestal fracture model by assuming a $400 \mathrm{D}$ permeability in the NS direction and $40 \mathrm{D}$ in the EW direction. (Orientations correspond to the expected principal directions of permeability in the pilot area.) The result was an elliptical steam zone (see Fig. 7), with an aspect ratio of 4 . After about six months the steam had travelled about $100 \mathrm{~m}$ in the NS direction and only $25 \mathrm{~m}$ in the perpendicular direction. Although the magnitude of anisotropy was chosen somewhat arbitrarily, this example does illustrate the potential impact that permeability anisotropy can have on the areal shape of the steam zone.

\section{DESIGN ISSUES}

Data gathering with new observation wells is essential for pilot interpretation, to plan future pilot extension and possible application in other fields. In this particular case, the locations of observation and injection wells will be first based on the work described above. We summarise the key points here for convenience:

1) There appear to be no clear benefits to be gained by lowering the oil rim before the steam injection.

2) Both injection wells should be confined to one local crest in order to provide a central point around which the steam zone can develop. Because the shape of the steam zone will be simpler and easier to predict, fewer observation wells are required for data gathering and for the interpretation of the pilot results.

3) The currently active production wells are located too far from the injectors to be used in the subsurface data gathering programme, although they will be used to monitor production rates and provide fluid samples for analysis.

4) It is critical that observation wells be placed close enough to the injection wells so that a response is seen within the time frame of the pilot in order to quantify the rate of accelerated gas-oil gravity drainage.
Injection-well and observation-well locations

Mainly on the basis of the simulation results that demonstrate a piston-like displacement of the steam zone (i.e. the slowest advance of the steam zone), the observation and injection wells can be located to ensure that the results of the pilot can be properly interpreted. A conceptual picture of the proposed pilot design is given in Fig. 8. As shown in the figure, both injection wells, denoted "INJ", are located at the top of one of the two local crests. Note that all well locations are still subject to optimisation with regards to surface obstructions. The observations wells, denoted "O1", "O2" and "O3" will have cased, large-diameter completions suitable for saturation and temperature logging.

The predicted advance of the steam zone is shown in the figure; together with the calculated arrival times indicated for each radius. To observe the advance of the steam zone within the first year of the steam pilot, observation well $\mathrm{O} 1$ will be positioned approximately $40 \mathrm{~m}$ from the injector. It will be located high in the structure, in order to measure cold and hot desaturation and to further verify the expected local piston-like displacement. Well $O 1$ will be located to maximise the permeability contrast with well $\mathrm{O} 2$ in order to study the effect of permeability anisotropy. The second observation well, $\mathrm{O} 2$, is to be positioned $180 \mathrm{~m}$ from the injector, further down structure, in a location where possible steam override can be monitored if it occurs. This observation well is planned north of the injector in the direction of the highest permeability.

It is expected that baseline (pre-pilot) comparisons of the desaturation profile as a function of depth from the two observation wells may provide both an indication of the level of desaturation due to primary production and further clues as to the degree of horizontal capillary continuity within the column. The capillary-pressure curves taken to date have been remarkably similar, even for samples from different cores and facies. If the capillary-pressure curves are indeed similar, then, for a reservoir in which all rock is in capillary contact (vertical and areal) the capillary forces can be expected to force the desaturation of the matrix to be strictly a function of subsea depth. However, without areal capillary contact, the reservoir will behave as a collection of independently draining matrix stacks.

In order to monitor the advance and extent of the steam zone as well as the local oil-rim thickness, another observation well $\mathrm{O} 3$ is to be drilled $100 \mathrm{~m} \mathrm{SE}$ from the injector, towards the production well. Further observation wells may be considered at a later date depending on the shape and nature of the steam zone's advance. 
OARN ALAM: STEAM ZONE ADVANTAGE \& PILOT DESIGN

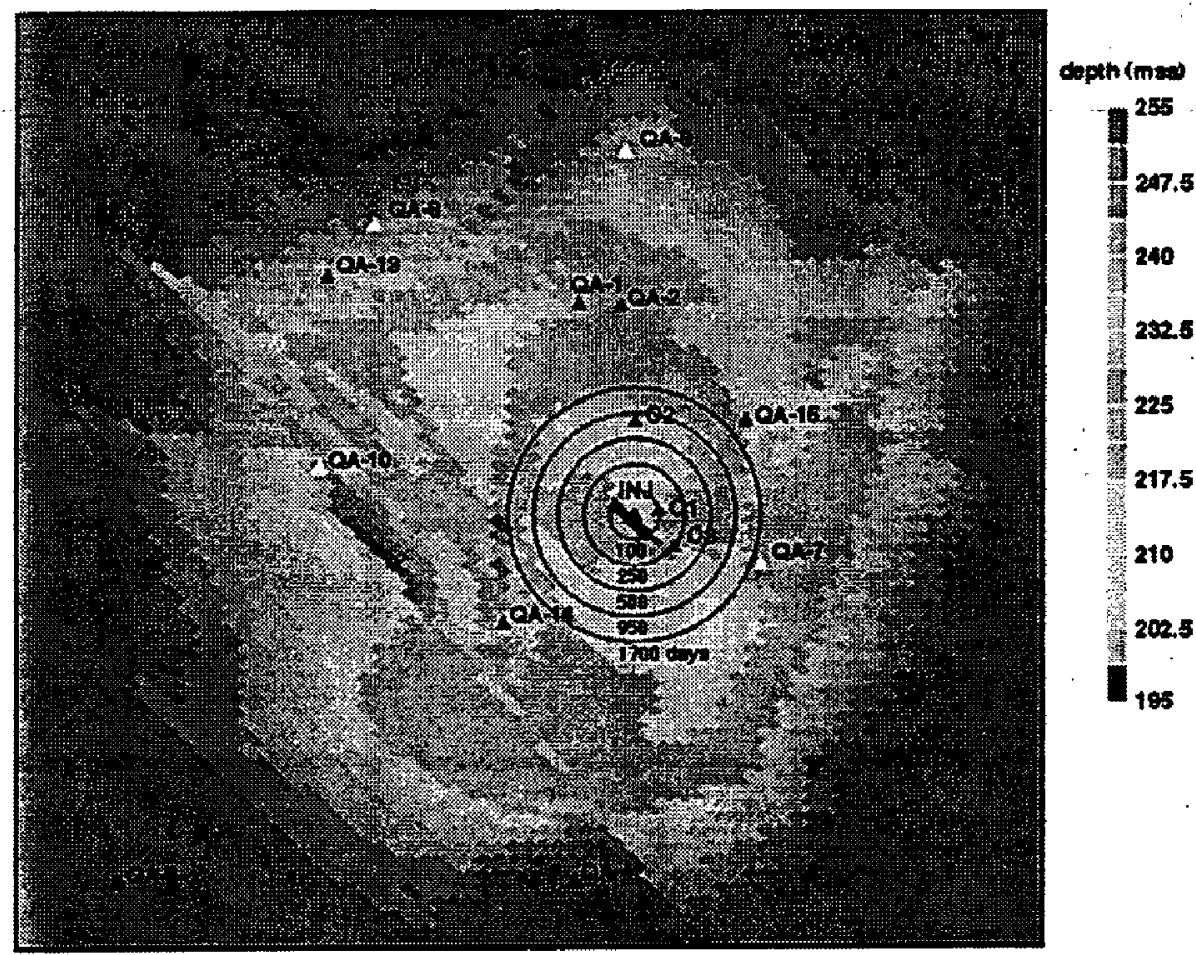

Figure 8 Conceptual picture of the proposed design of the steam pilot. The steam injector is located in the centre of the circles that indicate the radial advance of the steam for a pistonlike displacement; the arrival times of the steam zone at the different radii are indicated. The positions of the existing wells and observation wells $\mathrm{OI}_{1} \mathrm{O}_{2}$, and $\mathrm{O}_{3}$ are atso indicated.

\section{PILOT MONITORING}

The large-diameter observation wells are to be designed to accommodate a borehole gravimeter for accurate measurements of the matrix saturation. The observation wells will also be used to measure the arrival time of the steam zone and to deduce the nature of the displacement mechanism. Measurements will be taken before the start of the steam pilot to provide a baseline. Frequent logging of the temperature and saturation of the observation wells should reveal the desaturation caused by the thermally enhanced gravity-drainage rate from the matrix and the shape of the condensation front. In the case of piston-like displacement, the arrival time of the steam zone may also give an indication of the degree of fracture anisotropy.

To properly interpret the data from the steam pilot, it is also essential that the shape and position of the oil rim be monitored. The oil drained from the matrix will likely not be immediately captured by the producing wells and this will lead to an increase in the oil rim volume. Production logging, including monitoring of downhole temperature and compositional analysis of the produced fluids, is also planned. Lastly, the possibility of using seismic surveying or offset vertical seismic profiling (VSP) data to determine the extent of the heated zone in the reservoir is currently under consideration.

\section{DISCUSSION}

Different designs for the steam-injection pilot have been reviewed, and possible offtake scenarios have been considered. In particular, the influence of the main uncertainties on the oil production as well as on the fluid and the temperature distribution in the reservoir has been investigated.

The ultimate shape of the steam zone remains uncertain at this point, primarily because of the uncertainty in the reservoir fracture permeability. The steam pilot has been designed, and the locations of steam-injection wells and observation wells have been chosen under the assumption of a piston-like displacement, which is the case of the slowest (and most conservative) radial expansion of the steam zone.

\section{CONCLUSIONS}

\section{.}

1) Our simulation results for the pilot production response are in agreement with earlier predictions made with an analytical model, provided that the growth rate of the oil-rim volume is taken into 
account in the analytical model.

2) The steam-injection pilot should be confined to one local crest in order to confine the response to one area; otherwise, one might have to deal with essentially two separate pilots.

3) The oil rim should not be lowered prior to the pilot. Leaving the oil rim where it is will maximise the pilot response signal in observation wells.

4) Owing to the uncertainty in the effective fracture permeability $(1-1000 \quad \mathrm{D})$, the steam-gas displacement and therefore the shape of the steam zone remain uncertain. However, below an effective fracture permeability of $400 \mathrm{D}$, a piston-like displacement is expected.

5) Locations for injection and observation wells are conservatively based mainly on a piston-like displacement. Locations have been chosen so as to optimise production response and to quantify the contribution of gravity drainage to the production within the time period of the pilot.

6) Observation well logging as well as production logging is recommended to take place on a frequent basis.

7) Seismic surveying of the pilot area or the acquisition of VSP data will be considered as alternative means to monitor the heated area.

\section{ACKNOWLEDGEMENTS}

The authors express their thanks to the Ministry of Petroleum and Minerals of the Sultanate of Oman, Petroleum Development Oman and Shell Internationale Petroleum Maatschappij for their kind permission to allow the publication of this paper.

\section{SYMBOLS}

$r$ - radius of the steam zone

$\mathrm{t}$ - time in days

\section{REFERENCES}

[1] van Wunnik, J.N.M. and Wit, K.: "Improvement of Gravity Drainage by Steam Injection into a fissured reservoir: Analytical Evaluation", SPE/DOE 20251, 7th Symposium on Enhanced Oil Recovery, Tulsa 22-25 April 1990. 